\title{
Stand(ard) der allogenen Knochentransplantation
}

\author{
Ergebnisse einer Umfrage an Deutschen AO-Kliniken
}

\author{
H. Knaepler
}

\section{Zusammenfassung}

Die allogene Knochentransplantation ist ein in der Orthopädie und Unfallchirurgie häufig angewendetes Verfahren zur Füllung knöcherner Defekte. Sie wird seit ca. 50 Jahren angewendet, jährlich werden mindestens 500000 allogene Knochen weltweit transplantiert. Durch die Möglichkeit der Übertragung von Krankheitserregern mit dem allogenen Transplantat vom Spender auf den Empfänger sind jedoch strenge Richtlinien zu beachten. Die hier vorgestellte Umfrage an Deutschen AO-Kliniken sollte analysieren, inwieweit die allogene Knochentransplantation in diesen Kliniken standardisiert ist und die Richtlinien beachtet werden. Die Umfrageergebnisse zeigen große Unterschiede in den Indikationen für die Knochentransplantation und die Knochenbanktechnik. Eine einheitliche Standardisierung ist daher nicht feststellbar.

\section{Einleitung}

Die Notwendigkeit knöcherne Defekte zu füllen, besteht in ca. 15\% aller Operationen am Skelettsystem (4). Nach Etablierung der ersten Knochenbank 1950 durch Busch und Garber wurden knöcherne Defekte zunehmend durch allogene Knochentransplantate aufgefüllt (2).

In Deutschland wird mit einem jährlichen Bedarf von ca. 15000 -20000 allogenen Knochentransplantaten gerechnet.

In den USA rechnet man mit $300000-$ 400000 jährlichen Transplantationen $(5,11)$. Erst nach Bekanntwerden erster HIV-Übertragungen mit dem allogenen Knochen in den USA und später in Deutschland ist die Gefahr der allogenen Knochentransplantation ins breite Bewusstsein gelangt.

Prinzipiell können alle Krankheitserreger mit dem allogenen Knochentransplantat vom Spender auf den Empfänger übertragen werden $(3,9)$.

OP-JOURNAL 2000; 16: 290-292

(c) Georg Thieme Verlag Stuttgart · New York
Der wissenschaftliche Beirat der Bundesärztekammer hat von daher im August 1996 erstmals verbindliche Richtlinien zum Führen einer Knochenbank im Deutschen Ärzteblatt veröffentlicht, die sich im Wesentlichen an die amerikanischen Richtlinien der AATB halten (4). In diesen Richtlinien sind klare Vorgaben für die Auswahl der Knochenspender, den vorgeschriebenen Laboruntersuchungen, Untersuchungen des Explantates, Hinweise zur Lagerung der Explantate und zur Dokumentation gegeben. Die Richtlinien werden z.Z. einer Novellierung unterzogen. Ziel der jetzigen Umfrage war es herauszufinden, wie viele Knochenbanken an den Deutschen AO-Kliniken geführt werden und inwieweit diese den Richtlinien bzw. den vorgeschriebenen Standards entsprechen.

\section{Hauptteil}

Im August 1999 wurden 122 Fragebogen an deutsche unfallchirurgische und orthopädische AO-Kliniken ausgesandt. Außerdem 10 Fragebogen an die maxillofaziale Gruppe. Bis November 1999 haben 106 der 122 Unfallchirurgischen/Orthopädischen Kliniken geantwortet, dies entspricht einem Rücklauf von $87 \%$. 9 von 10 kieferchirurgischen Kliniken haben ebenfalls geantwortet.

\section{Ergebnisse der maxillofazialen Gruppe}

Keine der Kliniken betreibt eine Knochenbank, 7 von 9 Kliniken verwenden Ersatzmaterialien, 2 Kliniken haben hierzu keine Angaben gemacht.

\section{Ergebnisse unfallchirurgischer/ orthopädischer Kliniken}

Exakt die Hälfte der Kliniken, die geantwortet haben (53 Kliniken, 50\%) betreiben eine Knochenbank, die andere Hälfte entsprechend keine. Aufgeteilt in Orthopädisch-Unfallchirurgische Kliniken verschiebt sich diese Verteilung.

15 von 16 orthopädischen Kliniken (94\%) arbeiten mit einer allogenen Knochenbank, bei den unfallchirurgischen Kliniken sind dies 38 von 90, entsprechend $42 \%$.

Diejenigen Kliniken, die keine Knochenbank besitzen, verwenden neben der autogenen Spongiosa vielfältige Knochenersatzmaterialien bzw. teilweise auch industriell vertriebene humane Präparate, wobei in Tabelle 1 nur die häufigsten aufgeführt werden. Eine klare Linie oder Standardisierung ist hier nicht zu erkennen.

Auch bei der Lagerungstemperatur ergibt sich eine große Breite zwischen $+20^{\circ} \mathrm{C}$ und $-80^{\circ} \mathrm{C}$. Die vorgeschriebenen mindestens $-70^{\circ} \mathrm{C}$ werden jedoch von 44 der 53 Kliniken eingehalten. In der Lagerungsdauer entsprechen die Angaben zwischen 6 Monaten bis 5 Jahren den vorgeschriebenen Richtlinien.

Tab. 1 Nennung der häufigsten Alternativen zum allogenen Knochen

- autogene Spongiosa, Chips, Blöcke

- Endobon, Kalziumcarbonat, Hydroxylapatit, Biosorb, Cerasorb, BioOS, Synthacer

- Tutogen, DIZG 


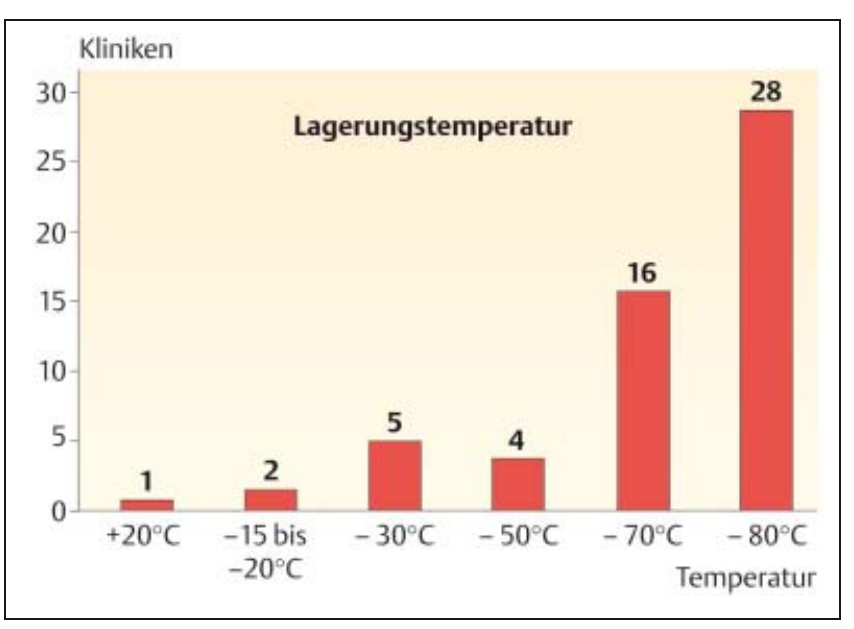

Abb. 1 Lagerungstemperaturen allogener Knochentransplantate.
Bei der Spenderserologie beachten 81\% die primäre Untersuchung des Spenders entsprechend den Richtlinien, jedoch nur $62 \%$ die vorgeschriebenen 3- bzw. 6-Monats-Tests.

Von den 23 Kliniken, die keine zweite serologische Spenderuntersuchung durchführen, verwenden 16 das thermische Desinfektionssystem Lobator SD 1/2. Dies entspricht den Richtlinien, da bei Verwendung dieses Systems auf den 2.HIV-Test verzichtet werden kann (4, Anmerkung 2 zu den Richtlinien).

4 Kliniken, die Autoklavieren führen keinerlei primäre oder sekundäre serologische Untersuchungen vom Spender durch. An Desinfektions- und Sterilisationsmaßnahmen wird im Wesentlichen das validierte Verfahren der $80^{\circ} \mathrm{C}$-Thermodesinfektion mit dem Lobator SD $1 / 2$ verwendet, 21 Kliniken benutzen dieses System. 10 Kliniken Autoklavieren, teilweise ohne Angaben der Temperatur und Zeitdauer.
In Tabelle 2 sind die am häufigsten genannten Indikationen und Kontraindikationen aufgeführt. Hier zeigt sich, dass für einige Kliniken eine Indikation zur allogenen Knochentransplantation gegeben ist, die für andere Kliniken eine absolute Kontraindikation darstellt.

\section{Diskussion und Schlussfolgerungen}

Als Hauptprobleme beim Führen einer Knochenbank wurden von den Kliniken genannt:

1. Organisation und Logistik der Knochenbank sind mit einem hohen Aufwand von Dokumentation, hohen Kosten und Lagerungsbedingungen verbunden.

2. Vermeintliche Unsicherheit in der rechtlichen Situation.

3. Die Unmöglichkeit der zweiten Spenderuntersuchung.

4. Die ausreichende Gewinnung geeigneter Transplantate, d.h. das Fehlen geeigneter oder ausreichender Spender in entsprechender Zahl.
5. Das Einbauverhalten großer Blöcke wurde als unsicher genannt.

6. Es wurde von den Kliniken teilweise angegeben, dass keine Richtlinien vorhanden wären.

\section{Die Analyse der Umfrageergebnisse muss zu folgenden Diskussionen Anlass geben}

Die allogene Knochentransplantation ist auch an den Deutschen AO-Kliniken ein weit verbreitetes Verfahren zur Füllung knöcherner Defekte.

Insbesondere in den orthopädischen Kliniken ist der Bedarf enorm hoch, insbesondere im Bereich der Wechseloperationen an Hüftgelenkprothesen. Bis auf eine orthopädische Klinik kam keine ohne eine Knochenbank aus. Aber auch die unfallchirurgischen Kliniken sind offensichtlich, zumindest zu $42 \%$, an den allogenen Knochen als Alternative gebunden.

Das Spektrum der Knochenersatzmaterialien ist entsprechend den von der Industrie angebotenen Produkten sehr groß, eine einheitliche Linie und Meinung ist nicht zu erkennen. Teilweise werden auch Präparate, insbesondere humanen Ursprungs von der Industrie angeboten, die keine entsprechende Zulassung als Arzneimittel besitzen.

Als alarmierend muss gewertet werden, dass einige Kliniken sich nicht an die verbindlichen Richtlinien zum Führen einer Knochenbank halten.

Eine Lagerungstemperatur $<70^{\circ} \mathrm{C}$ ist unzulässig, ebenso eine Unvollständigkeit der Spenderserologie im primären oder sekundären Test.

Besonders erschreckend ist dabei die Angabe, dass Kliniken, die Autoklavieren keinerlei Spenderuntersuchungen durchführen.

Dies geschieht offenbar in der falschen Annahme, dass durch die Autoklavierung alle Krankheitserreger sterilisiert werden. Dies entspricht nicht den Vorgaben, da die Autoklavierung in den Richtlinien nicht genannt wurde, weil es sich um ein nicht-validiertes Verfahren handelt. Bei dem im Operationssaal üblichen Autoklavieren, wie es zur Sterilisation chirurgischer Instrumente verwendet wird, werden zu kurze Einwirkzeiten verwendet, um einen größeren Knochenblock komplett zu autoklavieren, d.h. zu sterilisieren (8). große Knochendefekte

Tumoren

Gelenkfrakturen mit Defekten

Pseudarthrosen

frische Frakturen

Patient $>60$ Jahre
- ersatzschwaches Lager

kindliche Knochendefekte

Tumoren

Gelenkfrakturen

Pseudarthrosen

frische Frakturen

Patient $<50$ Jahre

keine 
Außerdem nimmt die Qualität des autoklavierten Knochens sowohl biomechanisch als auch in der biologischen Wertigkeit stark ab, so dass die Indikation angezweifelt werden muss (6).

Als einzig validiertes Verfahren werden von der Bundesärztekammer die thermische Inaktivierung oder Bestrahlung anerkannt.

Die Uneinheitlichkeit in der Bewertung allogener Knochentransplantate kommt am besten bei der Gegenüberstellung der Indikation und Kontraindikation zur Darstellung. Hier stehen Indikationen und Kontraindikationen in einer Spalte und zeigen die fehlende Standardisierung deutlich auf. Einigkeit besteht lediglich bei den Kontraindikationen im Bereich eines septischen oder ersatzschwachen Lagers bzw. bei offenen Frakturen. Aber auch hier wurden von einigen Kliniken generell keinerlei Kontraindikationen für allogene Knochentransplantate genannt. Die von den Kliniken genannten Hauptprobleme sind nur teilweise objektivierbar und zutreffend.

Ein wirkliches Hauptproblem stellt sicherlich die Organisation und Logistik der Knochenbank mit einem erheblichen Aufwand der Dokumentation dar. Diese Dokumentationspflicht wird in den nächsten Jahren noch zunehmen.

Erste Knochenbanken wurden in Deutschland bereits zertifiziert, im Jahr 2001 werden die ersten Seminare mit entsprechender Zertifizierung für Knochenbankbetreiber durchgeführt.

Die vermeintliche Unsicherheit in der rechtlichen Situation ist de facto nicht gegeben. Die Richtlinien und auch die Novellierung dieser Richtlinien geben klare Vorgaben, an denen man sich orientieren kann und muss.

Das Einbauverhalten größerer Blöcke ist durch mehrere große Studien untersucht worden (10). Hier wurde tierexperimentell und klinisch nachgewiesen, dass große allogene Blöcke teilweise Remodellierungs- und Revitalisierungszeiträume von mehreren Jahren brauchen. Dies muss bei entsprechender Belastung oder Metallentfernung bedacht werden.

Als Schlussfolgerungen bleibt aufgrund dieser Umfrage festzuhalten:

1. Eine einheitliche Meinung hinsichtlich der Wertigkeit, Notwendigkeit, Indikation und Kontraindikation allogener Knochentransplantate besteht nicht.
2. Eine Standardisierung in der Knochenbanktechnik ist nur teilweise gegeben.

3. Die Einhaltung der Richtlinien der Bundesärztekammer vom August 1996 geschieht nur unvollständig. Sie sind teilweise nicht bekannt, forensisch jedoch unabdingbar.

4. Die Vielfalt möglicher Alternativen (Knochenersatzmaterialien) ist kaum überschaubar und die biologische Wertigkeit wissenschaftlich bisher nur teilweise nachgewiesen.

\section{Zukünftige Planungen}

Interessanterweise haben $87 \%$ der AOMitglieder, die geantwortet haben, ihr Interesse an der Mitarbeit in der Arbeitsgruppe bekundet. Demzufolge ist das Interesse an der Mitarbeit höher als die prozentuale Beteiligung am Betreiben einer Knochenbank. Dies lässt sich so interpretieren, dass viele Kliniken eine Knochenbank betreiben wollen, aufgrund der o.a. Probleme jedoch zumindest bisher diese nicht oder nicht mehr durchführen. Es besteht die Notwendigkeit einer interdisziplinären Expertengruppe zur Informationsweitergabe, insbesondere hinsichtlich der rechtlichen Situation der Knochenbanken und der mikrobiologischen Problematik der Transplantate. Entsprechende Arbeitsgruppen sind inzwischen gebildet:

a) Arbeitskreis der Bundesärztekammer zur Novellierung der Richtlinien,

b) Arbeitskreis zur Etablierung regionaler Knochenbanken,

c) Arbeitskreis der deutschen AO,

d) Arbeitskreis der DGOT

Das Arztprivileg zur Betreibung lokaler Knochenbanken ( $\$ 80$ AMG, Abs. 2, 3 u. 4) besteht weiterhin.

Dies beinhaltet, dass wenn ein Knochentransplantat in einer Klinik entnommen wird und in der gleichen Klinik wieder transplantiert wird, es nicht als Arzneimittel gilt. Eine Weitergabe an andere Kliniken oder außerhalb der Klinik ist jedoch rechtlich nicht möglich.

Zur Weitergabe außerhalb der Klinik ist eine Herstellererlaubnis $(\S 13,14,15$ AMG) notwendig.

Der Knochen gilt dann als Arzneimittel. Es sind Bestrebungen im Gange, in Deutschland in den nächsten Jahren solche regionalen Knochenbanken aufzubauen, so dass auch kleinere Kliniken allogene Knochentransplantate verwenden können. Als Fernziel muss auch genannt werden, dass die Indikationsliste für allogene Knochentransplantate zumindest annähernd angeglichen oder vereinheitlicht werden muss.

Es dürften an Deutschen AO-Kliniken nur wenige Beispiele existieren, wo Indikation und Kontraindikationen für dasselbe Verfahren identisch sind.

Auch die Verwendung und Indikation der Knochenersatzmaterialien und deren biologische Einschätzung der Wertigkeit sind sicher ein weites Feld. Die Hersteller derartiger Knochenersatzmaterialien veröffentlichen bisweilen werbemäßig vermeintliche Tatsachen, die wissenschaftlich und rechtlich nicht dem gegenwärtigen Stand entsprechen.

\section{Literatur}

${ }^{1}$ American Association of Tissue Banks. Guidelines for banking of musculoskeletal tissues, AATB Newslett 3, p 2 (1979).

${ }^{2}$ Bush LF, Garber CZ. The bone bank, JAMA 1948; 588 - 594

${ }^{3}$ CDC, Transmission of HIV through bone transplantation: Case report and public health recommendations, MMWR 1988; 37: $597-599$

${ }^{4}$ Deutsches Ärzteblatt 1996; 93: Heft 34-35, A-2166-A-2171

${ }^{5}$ Knaepler H, Laubach S, Gotzen L. Die Knochenbank ein standardisiertes Verfahren? Ergebnisse einer bundesweiten Umfrage an chirurgischen Kliniken. Chirurg 1991; 61: $833-837$

${ }^{6}$ Knaepler H, v. Garrel, Seipp HM, Ascherl R, Gotzen L. Autoklavierung von allogenen Knochentransplantaten als Alternative zur konventionellen Knochenbank? Orthop. Praxis 1992; $18-22$

${ }^{7}$ Knaepler H, v. Garrel T. Sind die Risiken allogener Knochentransplantate noch vertretbar? Kongressband Der Deutschen Gesellschaft für Unfallchirurgie 1999; 93-97

${ }^{8}$ Metak G, Reeg S, Ascherl R, Gradinger R. Blümel. Nicht erreichte Sterilisationstemperatur in großen Knochentransplantaten eine Warnung bei der Verwendung autoklavierter Knochen. Zentraleuropäischer Unfallkongress 5,94 Budapest

${ }^{9}$ Schratt HE, Regel G, Lobenhoffer P, Tscherne H. Die Organisation einer Knochen- und Gewebebank, Unfallchirurg 1996; 99: 880 888

${ }^{10}$ Sangmeister M, Knaepler H, Ennis M, Kleinsorge F. Der allogene Knochenblock - Anwendungsprinzip und Behandlungsresultate. Hefte Unfallheilkd. 1998; 207: 447

11 Tomford WW. A perspective on bone banking in the United States. In: Bone Grafts, Derivates and Substitutes, M.R. Urist, B.T. O'Conner and R.G. Burwell, eds.; Butterwoth-Hejnemann, Oxford 1994

\section{Prof. Dr. med. H. Knaepler}

Chefarzt der Unfallchirurgischen Klinik

Klinikum Wetzlar-Braunfels

Forsthausstr. 1

35578 Wetzlar 